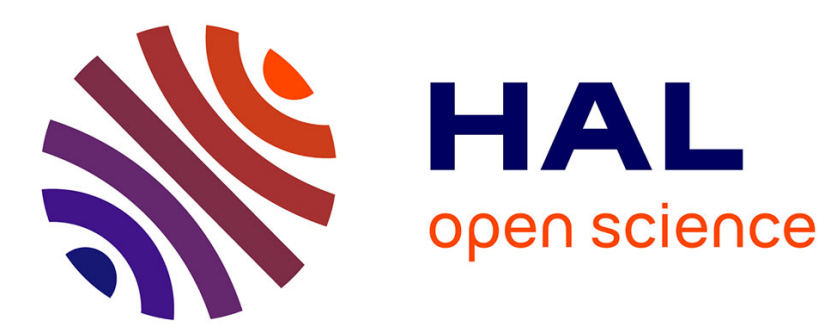

\title{
Second order statistics of bilinear forms of robust scatter estimators
}

\author{
Abla Kammoun, Romain Couillet, Frédéric Pascal
}

\section{To cite this version:}

Abla Kammoun, Romain Couillet, Frédéric Pascal. Second order statistics of bilinear forms of robust scatter estimators. 40th IEEE International Conference on Acoustics, Speech and Signal Processing (ICASSP 2015), Apr 2015, Brisbane, Australia. 10.1109/ICASSP.2015.7178604 . hal-01262625

\section{HAL Id: hal-01262625 \\ https://hal.science/hal-01262625}

Submitted on 26 Jan 2016

HAL is a multi-disciplinary open access archive for the deposit and dissemination of scientific research documents, whether they are published or not. The documents may come from teaching and research institutions in France or abroad, or from public or private research centers.
L'archive ouverte pluridisciplinaire HAL, est destinée au dépôt et à la diffusion de documents scientifiques de niveau recherche, publiés ou non, émanant des établissements d'enseignement et de recherche français ou étrangers, des laboratoires publics ou privés. 


\title{
SECOND ORDER STATISTICS OF BILINEAR FORMS OF ROBUST SCATTER ESTIMATORS
}

\author{
Abla Kammoun ${ }^{1}$, Romain Couillet ${ }^{2}$ and Frédéric Pascal ${ }^{2}$ \\ King Abdullah University of Science and Technology (KAUST), Saudi Arabia ${ }^{1}$ \\ Supélec, France ${ }^{2}$
}

\begin{abstract}
This paper lies in the lineage of recent works studying the asymptotic behaviour of robust-scatter estimators in the case where the number of observations and the dimension of the population covariance matrix grow at infinity with the same pace. In particular, we analyze the fluctuations of bilinear forms of the robust schrinkage estimator of covariance matrix. We show that this result can be leveraged in order to improve the design of robust detection methods. As an example, we provide an improved generalized likelihood ratio based detector which combines robustness to impulsive observations and optimality across the shrinkage parameter.
\end{abstract}

\section{INTRODUCTION}

Estimation of covariance matrices is a fundamental problem in statistical signal processing with numerous applications, most importantly in radar detection and estimation of direction of arrivals [1]. The commonly used estimator is the well-known sample covariance matrix (SCM) which coincides with the Maximum Likelihood estimator for Gaussian observations. If $\mathbf{x}_{1}, \cdots, \mathbf{x}_{n}$ denote the zeromean $n$ observations independent and identically distributed of size $N$, then the sample covariance estimator is given by:

$$
\hat{\mathbf{R}}=\frac{1}{n} \sum_{i=1}^{n} \mathbf{x}_{i} \mathbf{x}_{i}^{*}
$$

The popularity of the SCM owes to its low complexity and the existence of a good understanding of its behavior in the regimes $n \rightarrow \infty$ and $N$ fixed, and also that of $n, N$ tending to infinity with the same pace. In particular, it is already known that in the first regime, $\hat{\mathbf{R}}$ is a consistent estimator of the true covariance matrix of $\mathbf{x}_{i}$. Recent advances in random matrix theory have established that the SCM is no longer consistent when $N$ and $n$ are large and comparable, a regime which will be referred in the sequel to as $n, N \rightarrow \infty$. In general, a consistent estimate of the covariance matrix does not exist, except probably for special cases where an a priori structure of the covariance matrix is assumed. Hopefully, in practice, the object of interest is often not the covariance matrix of the observations but rather some scalar functional of it. The use of recent results from random matrix theory have allowed to devise consistent estimation techniques of these functionals in the regime $n, N \rightarrow \infty$. A wide range of applications have been considered ranging from source detection $[2,3]$ and subspace estimation methods in array processing [4] to performance metrics estimation in wireless communications [5, 6]. A common denominator of these methods is that they still fundamentally rely on the SCM, their consistency being obtained through a deep analysis of its asymptotic behaviour. Nevertheless, the use of SCM can be inadequate if some atypical observations exhibit an impulsive character, thereby inducing poor accuracy for finite $N$, $n$, even though asymptotically consistent. Such observations, often referred to as outliers can represent any impulsive source of noise, including high short-term interference in wireless networks [7] or clutter echoes in radar applications [8]. An alternative to the conventional use of SCM, which can be traced back to the early works of Huber [9] and Maronna [10] is constituted by the class of robust scatter estimators, which are known for their resilience to the presence of atypical observations. Among these estimators, we distinguish the Maronna's M-estimators given as the unique solution of:

$$
\hat{\mathbf{C}}_{N}=\frac{1}{n} \sum_{i=1}^{n} u\left(\frac{1}{N} \mathbf{x}_{i}^{*} \hat{\mathbf{C}}_{N}^{-1} \mathbf{x}_{i}\right) \mathbf{x}_{i} \mathbf{x}_{i}^{*}
$$

where $u$ (defined on $[0, \infty)$ ) is a positive function satisfying some appropriate conditions, and the Tyler M-estimator obtained by setting $u(x)=\frac{1}{x}$. Despite their confirmed resilience to impulsive noises, the performance study of robust estimation methods has essentially been performed under the classical regime $n \rightarrow \infty$ and $N$ fixed. It is only recently that a new wave of works has emerged which shed light on the asymptotic behaviour of robust scatter estimation methods in the regime $N, n \rightarrow \infty[11,12]$.

In this paper, we consider the robust shrinkage estimator proposed in [13] and given as the unique solution $\hat{\mathbf{C}}_{N}$ to:

$$
\hat{\mathbf{C}}_{N}(\rho)=(1-\rho) \frac{1}{n} \sum_{i=1}^{n} \frac{\mathbf{x}_{i} \mathbf{x}_{i}^{*}}{\frac{1}{N} \mathbf{x}_{i}^{*} \hat{\mathbf{C}}_{N}^{-1}(\rho) \mathbf{x}_{i}}+\rho \mathbf{I}_{N},
$$

where $\rho \in(\max \{0,(n-N) / n\}, 1]$. Such estimator is built upon the Tyler's M-estimate and upon the Ledoit-Woft shrinkage estimator [14]. It is particularly well-suited to scenarios where $N>n$, for which other estimators are badly conditioned if not undefined. In [11], it has been shown that $\sup _{\rho}\left\|\hat{\mathbf{C}}_{N}(\rho)-\hat{\mathbf{S}}_{N}(\rho)\right\| \stackrel{\text { a.s. }}{\longrightarrow} 0$ where $\hat{\mathbf{S}}_{N}(\rho)$ follows a classical random matrix model. The importance of this result lies in that it allows a better understanding of the behaviour of $\hat{\mathbf{C}}_{N}$ and more interestingly it facilitates the design of novel robust consistent estimation techniques. Such estimators are obtained using the convergence of $\hat{\mathbf{C}}_{N}(\rho)$ to $\hat{\mathbf{S}}_{N}(\rho)$. However, in some applications, where second order statistics are demanded, an understanding of the rate of convergence of $\hat{\mathbf{C}}_{N}(\rho)$ to $\hat{\mathbf{S}}_{N}(\rho)$ is essential for coming up with appropriate settings of the shrinkage parameter $\rho$. Of particular interest are quadratic forms of the type $\mathbf{a}^{*} \hat{\mathbf{C}}_{N}^{k} \mathbf{b}$ which naturally appears for $k=-1$ in the Generalized Likelihood Ratio detection technique for elliptical noise environments, often used in radar applications [8]. The study of the convergence rate of these quadratic forms is among the major contributions of this work. Contrary to first impressions (conveyed by the fact that $\left|\hat{\mathbf{C}}_{N}(\rho)-\hat{\mathbf{S}}_{N}(\rho)\right|$ seems to fluctuate at speed $N^{-\frac{1}{2}}$ ), we show that this rate is sufficient to ensure that $\mathbf{a}^{*} \hat{\mathbf{C}}_{N}^{k} \mathbf{b}$ and $\mathbf{a}^{*} \hat{\mathbf{S}}_{N}^{k} \mathbf{b}$ exhibit the 
same fluctuations. As an additional contribution, we show how this result can be exploited for the specific problem of signal detection in impulsive noise environments via the GLRT. In this context, we determine the shrinkage parameter $\rho$ which minimizes the probability of false detections and provide an empirical consistent estimate of this parameter. Finally, we conclude by providing simulation results that support our theoretical findings.

\section{MAIN RESULTS}

We consider $\mathbf{x}_{1}, \cdots, \mathbf{x}_{n}$ be $n$ zero mean independent observations of $\mathbb{C}^{N}$, which satisfy:

$$
\mathbf{x}_{i}=\sqrt{\tau_{i}} \mathbf{A}_{N} \mathbf{w}_{i}
$$

where $\mathbf{w}_{i} \in \mathbb{C}^{N}$ are Gaussian zero mean random vectors with covariance $\mathbf{I}_{N}$ and $\tau_{i}>0$ are random or deterministic scalars. Note that letting $\tau_{i}=\frac{\tilde{\tau}_{i}}{\left\|\mathbf{w}_{i}\right\|}$ for some $\tilde{\tau}_{i}$ independent of $\mathbf{w}_{i}$, vectors $\mathbf{x}_{i}$ (2) belongs to the class of elliptically distributed random vectors. Denote $\mathbf{C}_{N}=\mathbf{A}_{N} \mathbf{A}_{N}^{*} \in \mathbb{C}^{N \times N}$ such that $\nu_{N} \triangleq$ $\frac{1}{N} \sum_{i=1}^{N} \delta_{\lambda_{i}\left(\mathbf{C}_{N}\right)} \rightarrow \nu$ weakly, with $\lim \sup \left\|\mathbf{C}_{N}\right\|<\infty$ and $\frac{1}{N} \operatorname{tr} \mathbf{C}_{N}=1$.

In this work, we consider the regime where $N, n \rightarrow \infty$ and $c_{N} \triangleq \frac{N}{n} \rightarrow c \in(0, \infty)$, and propose to analyze under this regime the behaviour of the robust-scatter estimator of [13] (See eq. 1).

The first-order asymptotic behaviour of (1) has been studied in [11]. Before presenting our second-order result, we shall review the main findings of [11], in order to facilitate the understanding of our work.

\subsection{First order result}

The difficulty of analyzing the asymptotic behaviour of the robustscatter estimator lies in the rank-1 matrices involved in the sum of (1) being dependent through $\hat{\mathbf{C}}_{N}(\rho)$. At first glance, this observation might make us think that the asymptotic analysis of $\hat{\mathbf{C}}_{N}(\rho)$ is out of the framework of the theory of large random matrices. However, a careful investigation of the expression of $\hat{\mathbf{C}}_{N}(\rho)$ can lead to replace it by a more suitable asymptotic random equivalent for which many results from random matrix theory can easily apply. A thorough proof of the asymptotic behaviour can be found in [11]. Hereafter, we develop heuristics that show how one can obtain a more tractable random equivalent of $\hat{\mathbf{C}}_{N}(\rho)$.

For that, consider $\hat{\mathbf{C}}_{(i)}$ the matrix obtained by removing the outer product involving $\mathbf{x}_{i}$, i.e, $\hat{\mathbf{C}}_{(i)}=\hat{\mathbf{C}}_{N}(\rho)-\frac{1-\rho}{n} \frac{\mathbf{x}_{i} \mathbf{x}_{i}^{*}}{\frac{1}{N} \mathbf{x}_{i}^{*} \hat{\mathbf{C}}_{N}^{-1}(\rho) \mathbf{x}_{i}}$. Using the relation $\left(\mathbf{B}-t \mathbf{v} \mathbf{v}^{*}\right)^{-1} \mathbf{v}=\mathbf{B}^{-1} \mathbf{v} /\left(1+t \mathbf{v}^{*} \mathbf{B}^{-1} \mathbf{v}\right)$, we get:

$$
\mathbf{z}_{i}^{*} \mathbf{C}_{N}^{-1}(\rho) \mathbf{z}_{i}=\left(1+(1-\rho) c_{N}\right) \mathbf{z}_{i}^{*} \mathbf{C}_{(i)}^{-1} \mathbf{z}_{i}
$$

Hence, (1) becomes:

$$
\hat{\mathbf{C}}_{N}(\rho)=\frac{(1-\rho)}{1-(1-\rho) c_{N}} \frac{1}{n} \sum_{i=1}^{n} \frac{\mathbf{z}_{i} \mathbf{z}_{i}^{*}}{\frac{1}{N} \mathbf{z}_{i}^{*} \hat{\mathbf{C}}_{(i)}^{-1} \mathbf{z}_{i}}+\rho \mathbf{I}_{N} .
$$

One can easily convince oneself that the dependence of $\hat{\mathbf{C}}_{(i)}$ on $\mathbf{z}_{i}$ is quite weak. Thus, the asymptotic limit of $d_{i}(\rho) \triangleq \frac{1}{N} \mathbf{z}_{i}^{*} \hat{\mathbf{C}}_{(i)}^{-1} \mathbf{z}_{i}$ is the same as the one of $\frac{1}{N} \operatorname{tr} \mathbf{C}_{N} \hat{\mathbf{C}}_{(i)}^{-1}$ using the trace lemma in [15, Lemma B.26] and also that of $\frac{1}{N} \operatorname{tr} \mathbf{C}_{N} \hat{\mathbf{C}}_{N}^{-1}(\rho)$ by rank-1 perturbation arguments. In light of this intuition, if $\gamma_{N}(\rho)$ denotes the asymptotic limits of all the $d_{i} \mathrm{~s}$ (the limit of all the $d_{i} \mathrm{~s}$ is the same), then:

$$
\hat{\mathbf{C}}_{N}(\rho) \simeq \frac{(1-\rho)}{1-(1-\rho) c_{N}} \frac{1}{n} \sum_{i=1}^{n} \frac{\mathbf{z}_{i} \mathbf{z}_{i}^{*}}{\gamma_{N}(\rho)}+\rho \mathbf{I}_{N}
$$

Therefore, taking the trace of the inverse of (3) times $\mathbf{C}_{N}$,

$$
\gamma_{N}(\rho) \simeq \frac{1}{N} \operatorname{tr} \hat{\mathbf{C}}_{N}\left(\frac{(1-\rho)}{1-(1-\rho) c_{N}} \frac{1}{n} \sum_{i=1}^{n} \frac{\mathbf{z}_{i} \mathbf{z}_{i}^{*}}{\gamma_{N}(\rho)}+\rho \mathbf{I}_{N}\right)^{-1}
$$

The behaviour of the matrix inverse in the right-hand side of (4) is well-studied by the theory of large random matrices. Its trace can be handled using standard kind of calculations thereby implying, with a slight abuse of the notations, that $\gamma_{N}$ is the unique solution to:

$$
\gamma_{N}(\rho)=\int \frac{t}{\gamma_{N}(\rho) \rho+(1-\rho) t} \nu_{N}(d t)
$$

From this intuition, we have the following approximation matrix for $\hat{\mathbf{C}}_{N}$ :

$$
\hat{\mathbf{S}}_{N}(\rho)=\frac{1}{\gamma_{N}(\rho)} \frac{1-\rho}{1-(1-\rho) c_{N}} \frac{1}{n} \sum_{i=1}^{n} \mathbf{z}_{i} \mathbf{z}_{i}^{*}+\rho \mathbf{I}_{N}
$$

We recall now the main steps of the rigorous proof of this equivalence. Let $f_{i}=\frac{d_{i}(\rho)}{\gamma_{N}(\rho)}$ and assume that $f_{1} \leq \cdots \leq f_{n}$. The work of [11] establishes that for any $\ell>0$ and any sequence $\rho_{n} \in \mathcal{R}_{\kappa} \triangleq$ $\left[\kappa+\max \left(0,1-c^{-1}, 1\right)\right]$ with $\kappa>0, f_{1} \geq 1-\ell$ and $f_{n} \leq 1+\ell$. The proof is done by contradiction. We will just provide the main milestones for the control of $f_{n}$. We start by upper-bounding $d_{n}(\rho)$ by:

$$
\begin{aligned}
d_{n}(\rho) & \leq \frac{1}{N} \mathbf{z}_{n}^{*}\left(\frac{1-\rho}{1-(1-\rho) c_{N}} \frac{1}{n} \sum_{i=1}^{n} \frac{\mathbf{z}_{i} \mathbf{z}_{i}^{*}}{d_{n}(\rho)}+\rho \mathbf{I}_{N}\right)^{-1} \mathbf{z}_{n} \\
& \leq \frac{d_{n}(\rho)}{N} \mathbf{z}_{n}^{*}\left(\frac{1-\rho}{1-(1-\rho) c_{N}} \frac{1}{n} \sum_{i=1}^{n} \mathbf{z}_{i} \mathbf{z}_{i}^{*}+d_{n}(\rho) \rho \mathbf{I}_{N}\right)^{-1} \mathbf{z}_{n}
\end{aligned}
$$

If there exists a sequence $\rho_{n} \in \mathcal{R}_{\kappa}$ over which $f_{n}\left(\rho_{n}\right) \geq 1+\ell$, then:

$1 \leq \frac{1}{N} \mathbf{z}_{n}^{*}\left(\frac{1-\rho}{1-(1-\rho) c_{N}} \frac{1}{n} \sum_{i=1}^{n} \mathbf{z}_{i} \mathbf{z}_{i}^{*}+\left(\gamma\left(\rho_{n}\right)+\ell\right) \rho_{n} \mathbf{I}_{N}\right)^{-1} \mathbf{z}_{n}$

The quadratic form in the right-hand side of turns out to converge to a limit which is strictly less than 1 , thereby leading to a contradiction with the above inequality. We then have,

$$
\sup _{\rho \in \mathcal{R}_{\kappa}} \max _{1 \leq i \leq n}\left|d_{i}(\rho)-\gamma_{N}(\rho)\right| \stackrel{\text { a.s. }}{\longrightarrow} 0
$$

which therefore leads to the following first-order result:

Theorem 1. Consider the regime where $N, n \rightarrow \infty$ and $c_{N} \rightarrow c$. For any $\kappa>0$ small, define $\mathcal{R}_{\kappa} \triangleq\left[\kappa+\max \left(0,1-c^{-1}, 1\right)\right]$. Then,

$$
\sup _{\rho \in \mathcal{R}_{\kappa}}\left\|\hat{\mathbf{C}}_{N}(\rho)-\hat{\mathbf{S}}_{N}(\rho)\right\| \stackrel{\text { a.s. }}{\longrightarrow} 0 .
$$




\subsection{Second order result}

In this section, we analyse the fluctuations of quadratic forms of the type $\mathbf{a}^{*} \hat{\mathbf{C}}_{N}^{k} \mathbf{b}$. From the most recent works studying the fluctuations of random quadratic forms [16], one can intuitively state that the convergence rate of $\mathbf{a}^{*} \hat{\mathbf{C}}_{N}^{k} \mathbf{b}$ is of order $N^{-\frac{1}{2}}$. In order to transpose the fluctuations of $\mathbf{a}^{*} \hat{\mathbf{C}}_{N}^{k} \mathbf{b}$ to that of $\mathbf{a}^{*} \hat{\mathbf{S}}_{N}^{k} \mathbf{b}$, we need to establish that the difference $\mathbf{a}^{*} \hat{\mathbf{C}}_{N}^{k} \mathbf{b}-\mathbf{a}^{*} \hat{\mathbf{S}}_{N}^{k} \mathbf{b}$ converges to zero at a rate less than $N^{-\frac{1}{2}}$. A careful analysis of the above sketch of the proof reveals that a futher improvement of the first order result is possible by considering $N^{\frac{1}{2}-\epsilon} f_{i}$ for $\epsilon>0$ instead of $f_{i}$. The contradiction will still hold since the convergence speed of the quadratic form (5) is of order $N^{-\frac{1}{2}}$. Under the same setting of Theorem 1, we therefore have:

$$
\sup _{\rho \in \mathcal{R}_{\kappa}} N^{\frac{1}{2}-\epsilon}\left\|\hat{\mathbf{C}}_{N}(\rho)-\hat{\mathbf{S}}_{N}(\rho)\right\| \stackrel{\text { a.s. }}{\longrightarrow} 0
$$

which is not sufficient in view of the above discussion. In our opinion, the convergence speed in (6) cannot be improved to $N^{-\frac{1}{2}}$. Nonetheless, by virtue of an averaging effect, the fluctuation of special forms of functionals $\hat{\mathbf{C}}_{N}(\rho)$ can be further improved. Although linear functionals of the eigenvalues could have been considered, we focus our attention in this work on bilinear forms of the type $\mathbf{a}^{*} \hat{\mathbf{C}}_{N}^{k}(\rho) \mathbf{b}$ where $\mathbf{a}$ and $\mathbf{b}$ are unit norm vectors in $\mathbb{C}^{N}$ and $k \in \mathbb{Z}$. In particular, we prove in this work the following result:

Theorem 2. Under the setting of Theorem 1, for any $\epsilon>0$ and every $k \in \mathbb{Z}$ :

$$
\sup _{\rho \in \mathcal{R}_{\kappa}} N^{1-\epsilon}\left|\mathbf{a}^{*} \hat{\mathbf{C}}_{N}^{k}(\rho) \mathbf{b}-\mathbf{a}^{*} \hat{\mathbf{S}}_{N}^{k}(\rho) \mathbf{b}\right| \stackrel{\text { a.s. }}{\longrightarrow} 0 .
$$

An immediate consequence of Theorem 2 is that fluctuations of bilinear forms of the type $\mathbf{a}^{*} \hat{\mathbf{C}}_{N}^{k}(\rho) \mathbf{b}$ are the same as those of $\mathbf{a}^{*} \hat{\mathbf{S}}_{N}^{k}(\rho) \mathbf{b}$. This is because the convergence rate of the difference $\mathbf{a}^{*} \hat{\mathbf{C}}_{N}^{k}(\rho) \mathbf{b}-\mathbf{a}^{*} \hat{\mathbf{S}}_{N}^{k}(\rho) \mathbf{b}$ is less than $N^{-\frac{1}{2}}$. The interest of this finding becomes even more obvious when we notice that $\mathbf{a}^{*} \hat{\mathbf{S}}_{N}^{k}(\rho) \mathbf{b}$ are classical objects in random matrix theory for which an important load of new results exist. More formally, we state the following corollary:

Corollary 1. Assume that under the regime $N, n \rightarrow \infty$, there exists $\sigma_{N}^{2}>0$ such that:

$$
\sqrt{\frac{N}{\sigma_{N}^{2}}}\left(\mathbf{a}^{*} \hat{\mathbf{S}}_{N}^{k}(\rho) \mathbf{b}-\mathbb{E} \mathbf{a}^{*} \hat{\mathbf{S}}_{N}^{k}(\rho) \mathbf{b}\right) \underset{N, n \rightarrow \infty}{\stackrel{\mathcal{D}}{\longrightarrow}} \mathcal{N}(0,1)
$$

Then,

$$
\sqrt{\frac{N}{\sigma_{N}^{2}}}\left(\mathbf{a}^{*} \hat{\mathbf{C}}_{N}^{k}(\rho) \mathbf{b}-\mathbb{E} \mathbf{a}^{*} \hat{\mathbf{C}}_{N}^{k}(\rho) \mathbf{b}\right) \underset{N, n \rightarrow \infty}{\stackrel{\mathcal{D}}{\longrightarrow}} \mathcal{N}(0,1)
$$

Sketch of the proof of Theorem 2. Similar to the first order results, the main difficulty is on the control of quantities $d_{i}(\rho)$ which are dependent and do not admit closed-form expressions. However, the same methodology cannot be pursued, mainly because replacing quantities $d_{i}(\rho)$ by $\gamma_{N}(\rho)$ will not be sufficient for the second-order result to hold, the difference $\gamma_{N}(\rho)-d_{i}(\rho)$ being of order $O\left(N^{-\frac{1}{2}}\right)$. We need thus a refined approximation of $d_{i}(\rho)$ which has to be at the same time tractable and closer to $d_{i}(\rho)$ than is $\gamma_{N}(\rho)$. Recall that $d_{i}(\rho)=\frac{1}{N} \mathbf{z}_{i}^{*} \hat{\mathbf{C}}_{(i)}^{-1}(\rho) \mathbf{z}_{i}$. A natural random approximation of $d_{i}(\rho)$ would be obtained by substituting $\hat{\mathbf{C}}_{(i)}^{-1}(\rho)$ by $\hat{\mathbf{S}}_{(i)}^{-1}(\rho)$ with:

$$
\hat{\mathbf{S}}_{(i)}=\hat{\mathbf{S}}_{N}-\frac{1}{\gamma_{N}(\rho)} \frac{1-\rho}{1-(1-\rho) c_{N}} \frac{1}{n} \mathbf{z}_{i} \mathbf{z}_{i}^{*}
$$

thereby yielding:

$$
\tilde{d}_{i}=\frac{1}{N} \mathbf{z}_{i}^{*} \hat{\mathbf{S}}_{(i)}^{-1}(\rho) \mathbf{z}_{i}
$$

Using a proof by contradiction similar to the one in the first order result, we can establish that:

$$
N^{1-\epsilon} \sup _{\rho \in \mathcal{R}_{\kappa}} \max _{1 \leq i \leq n}\left|\tilde{d}_{i}(\rho)-d_{i}(\rho)\right| \stackrel{\text { a.s. }}{\longrightarrow} 0 .
$$

If we define $\tilde{\mathbf{S}}_{N}(\rho)=\frac{1-\rho}{1-(1-\rho) c_{N}} \frac{1}{n} \sum_{i=1}^{n} \frac{\mathbf{z}_{i} \mathbf{z}_{i}^{*}}{\tilde{d}_{i}(\rho)}+\rho \mathbf{I}_{N}$, we therefore have:

$$
\sup _{\rho \in \mathcal{R}_{\kappa}} N^{1-\epsilon}\left|\mathbf{a}^{*} \hat{\mathbf{C}}_{N}^{k}(\rho) \mathbf{b}-\mathbf{a}^{*} \tilde{\mathbf{S}}_{N}^{k}(\rho) \mathbf{b}\right| \stackrel{\text { a.s. }}{\longrightarrow} 0 .
$$

The problem is thus reduced to showing:

$$
\sup _{\rho \in \mathcal{R}_{\kappa}} N^{1-\epsilon}\left|\mathbf{a}^{*} \tilde{\mathbf{S}}_{N}^{k}(\rho) \mathbf{b}-\mathbf{a}^{*} \hat{\mathbf{S}}_{N}^{k}(\rho) \mathbf{b}\right| \stackrel{\text { a.s. }}{\longrightarrow} 0,
$$

a quantity which does not depend anymore on the less intractable random variables $d_{i}(\rho)$. The proof of (7) is however quite involved and relies on the use of standard but very technical tools of random matrix theory.

\section{APPLICATION TO THE GLRT DETECTOR}

We provide here an application to the detection of a known $N$-variate signal embedded in impulsive noise. The detection of the signal of interest consists in deciding between the following hypothesis:

$$
\mathbf{y}= \begin{cases}\mathbf{x}, & H_{0} \\ \alpha \mathbf{p}+\mathbf{x}, & H_{1}\end{cases}
$$

where $\alpha>0$ accounts for the unknown propagation attenuation and $\mathbf{x}$ follows the same model described in (2). In order to estimate the covariance of the noise $\mathbf{C}_{N}$, we assume that we have already collected $n$ observations corresponding to purely noise signals. We consider the robust-scatter estimate $\hat{\mathbf{C}}_{N}(\rho)$ in (1). The GLRT corresponding to the above hypothesis testing [8] reads:

$$
T_{N}(\rho) \underset{\mathcal{H}_{0}}{\stackrel{\mathcal{H}_{1}}{\gtrless}} \Gamma
$$

for some detection threshold $\Gamma$ where

$$
T_{N}(\rho) \triangleq \frac{\left|\mathbf{y}^{*} \hat{\mathbf{C}}_{N}^{-1}(\rho) \mathbf{p}\right|}{\sqrt{\mathbf{y}^{*} \hat{\mathbf{C}}_{N}^{-1}(\rho) \mathbf{y}} \sqrt{\mathbf{p}^{*} \hat{\mathbf{C}}_{N}^{-1}(\rho) \mathbf{p}}} .
$$

Our objective is to characterize the false alarm probability of the detector. That is, under $H_{0}$ (i.e., $\mathbf{y}=\mathbf{x}$ ), we shall evaluate the probability $P\left(T_{N}(\rho)>\Gamma\right)$. Since for fixed $\Gamma$, it appears that $T_{N}(\rho) \stackrel{\text { a.s. }}{\longrightarrow}$ 0 and every $\rho$, which does not say much about the actual test performance, we rather consider the case where $\Gamma=N^{-\frac{1}{2}} t$ for some $t>0$. In this case, our objective is to characterize the false alarm probability:

$$
P\left(T_{N}(\rho)>\frac{t}{\sqrt{N}}\right)
$$

Based on the result of Theorem 2 we prove the following: 
Theorem 3. For $z \in \mathbb{R}^{-}$, let $m(z)$ be the unique real positive solution to:

$$
m(z)=\left(-z+c \int \frac{(1-\rho) x}{1+(1-\rho m(z)) x} \nu(d x)\right)^{-1}
$$

Let $N, n \rightarrow \infty$ with $c_{N} \rightarrow c \in(0, \infty)$. Then,

$$
\sup _{\rho \in \mathcal{R}_{\kappa}}\left|P\left(T_{N}(\rho)>\frac{t}{\sqrt{N}}\right)-\exp \left(-\frac{t^{2}}{2 \sigma_{N}^{2}(\underline{\rho})}\right)\right| \rightarrow 0
$$

where $\underline{\rho}=\frac{\rho}{\rho+\frac{1}{\gamma_{N}(\rho)} \frac{1-\rho}{1-(1-\rho) c}}$ and:

$$
\begin{aligned}
\sigma_{N}^{2}(\underline{\rho}) & =\frac{\frac{1}{2} \mathbf{p}^{*} \mathbf{C}_{N} \mathbf{Q}_{N}^{2}(\underline{\rho}) \mathbf{p}}{\mathbf{p}^{*} \mathbf{Q}_{N}(\underline{\rho}) \mathbf{p} \frac{1}{N} \operatorname{tr} \mathbf{C}_{N} \mathbf{Q}_{N}(\underline{\rho})} \\
& \times \frac{1}{\left(1-c(1-\rho)^{2} m(-\underline{\rho})^{2} \frac{1}{N} \operatorname{tr} \mathbf{C}_{N}^{2} \mathbf{Q}_{N}^{2}(\underline{\rho})\right)}
\end{aligned}
$$

with $\mathbf{Q}_{N}(\underline{\rho}) \triangleq\left(\mathbf{I}_{N}+(1-\underline{\rho}) m(-\underline{\rho}) \mathbf{C}_{N}\right)^{-1}$

The result of theorem 3 provides an analytical characterization of the performance of the GLRT for each $\rho$ which suggests in particular the existence of values for $\rho$ which minimize the false alarm probability for given $t>0$. Note, in passing, that, independently of $t$, minimizing the false alarm rate is asymptotically equivalent to minimizing $\sigma_{N}^{2}(\rho)$ over $\rho$. However, the expression of $\sigma_{N}^{2}(\rho)$ depends on the covariance matrix $\mathbf{C}_{N}$ which is unknown and therefore does not allow for an immediate online choice of $\rho$. To handle this problem, the following proposition provides a consistent estimate for $\sigma_{N}^{2}(\underline{\rho})$ based on $\hat{\mathbf{C}}_{N}(\rho)$ and $\mathbf{p}$ :

Proposition 1 (Empirical performance estimation). For $\rho \in$ $\left(\max \left\{0,1-c_{N}^{-1}\right\}, 1\right)$ and $\underline{\rho}$ defined as above, let $\hat{\sigma}_{N}^{2}(\underline{\rho})$ be given by

$$
\begin{aligned}
\hat{\sigma}_{N}^{2}(\underline{\rho}) & \triangleq \frac{1}{2} \frac{1-\underline{\rho} \cdot \frac{\mathbf{p}^{*} \hat{\mathbf{C}}_{N}^{-2}(\rho) \mathbf{p}}{\mathbf{p}^{*} \hat{\mathbf{C}}_{N}^{-1}(\rho) \mathbf{p}} \cdot \frac{1}{N} \operatorname{tr} \hat{\mathbf{C}}_{N}(\rho)}{\left(1-c+c \underline{\rho} \frac{1}{N} \operatorname{tr} \hat{\mathbf{C}}_{N}^{-1}(\rho) \cdot \frac{1}{N} \operatorname{tr} \hat{\mathbf{C}}_{N}(\rho)\right)} \\
& \times \frac{1}{\left(1-\underline{\rho} \frac{1}{N} \operatorname{tr} \hat{\mathbf{C}}_{N}^{-1}(\rho) \cdot \frac{1}{N} \operatorname{tr} \hat{\mathbf{C}}_{N}(\rho)\right)}
\end{aligned}
$$

Also let $\hat{\sigma}_{N}^{2}(1) \triangleq \lim _{\underline{\rho} \uparrow 1} \hat{\sigma}_{N}^{2}(\underline{\rho})$. Then we have

$$
\sup _{\rho \in \mathcal{R}_{\kappa}}\left|\sigma_{N}^{2}(\underline{\rho})-\hat{\sigma}_{N}^{2}(\underline{\rho})\right| \stackrel{\text { a.s. }}{\longrightarrow} 0 \text {. }
$$

Since both the estimation of $\sigma_{N}^{2}(\rho)$ in Proposition 1 and the convergence in Theorem 3 are uniform over $\rho \in \mathcal{R}_{\kappa}$, we have the following result.

Corollary 2 (Empirical performance optimum). Let $\hat{\sigma}_{N}^{2}(\rho)$ be defined as in Proposition 1 and define $\hat{\rho}_{N}^{*}$ as any value satisfying

$$
\hat{\rho}_{N}^{*} \in \operatorname{argmin}_{\rho \in \mathcal{R}_{\kappa}}\left\{\hat{\sigma}_{N}^{2}(\underline{\rho})\right\}
$$

(this set being in general a singleton). Then, for every $t>0$,

$$
P\left(\sqrt{N} T_{N}\left(\hat{\rho}_{N}^{*}\right)>t\right)-\inf _{\rho \in \mathcal{R}_{\kappa}}\left\{P\left(\sqrt{N} T_{N}(\rho)>t\right)\right\} \rightarrow 0 .
$$

\section{NUMERICAL RESULTS}

In this section, we assess the accuracy of our theoretical results by means of simulations. In particular, we show in Fig 1 that as predicted by Theorem 3, the statistic $T_{N}(\rho)$ is uniformly wellapproximated by a Rayleigh distribution random variable $R_{N}(\underline{\rho})$ with parameter $\sigma_{N}(\rho)$. We note that good approximations are achieved although the number of sensors and of observations are far from the asymptotic regime. In a second experiment, we jus-

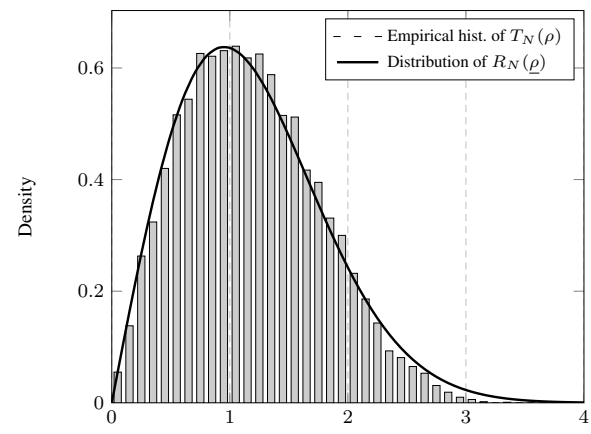

Fig. 1. Histogram distribution function of the $\sqrt{N} T_{N}(\rho)$ versus $R_{N}(\underline{\rho}), N=20, p=N^{-\frac{1}{2}}[1, \ldots, 1]^{\mathrm{T}},\left[\mathbf{C}_{N}\right]_{i j}=0.7^{|i-j|}$, $c_{N}=1 / 2, \rho=0.2$.

tify the applicability of using $\hat{\rho}_{N}^{*}$. For that, we represent in Fig. 2 false alarm rates of the detector $P\left(T_{N}\left(\hat{\rho}_{N}^{*}\right)>\Gamma\right)$ with respect to the threshold $\Gamma=t / \sqrt{N}$ for $N=20$ and $N=100$, along with its asymptotic approximations $\exp \left(-t^{2} /\left(2 \sigma_{N}^{* 2}\right)\right.$ where $\sigma_{N}^{* 2}$ is the minimizer of $\sigma_{N}^{2}(\underline{\rho})$. These rates are numerically obtained out of $10^{6}$ Monte Carlo simulations. This figure shows that even errors of order $10^{-4}$ are well-approximated for large $N$, while only errors of order $10^{-2}$ can be evaluated for small $N$.

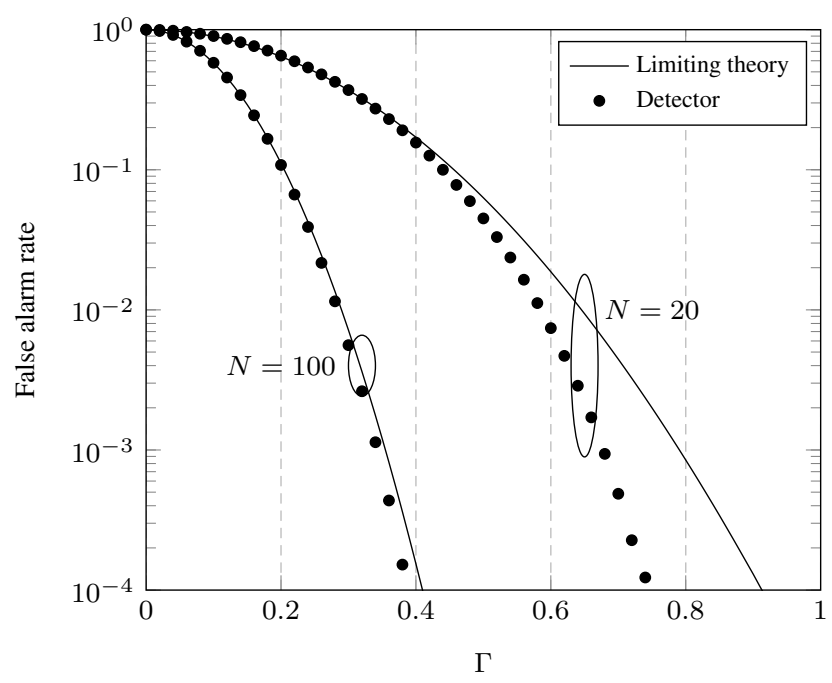

Fig. 2. False alarm rate $P\left(T_{N}\left(\hat{\rho}_{N}^{*}\right)>\Gamma\right)$ for $N=20$ and $N=100$, $p=N^{-\frac{1}{2}}[1, \ldots, 1]^{\mathrm{T}},\left[\mathbf{C}_{N}\right]_{i j}=0.7^{|i-j|}, c_{N}=1 / 2$. 


\section{REFERENCES}

[1] E. Ollila, D. E. Tyler, V. Koivunen, and H. V. Poor, "Complex Elliptically Symmetric Distributions: Survey, New Results and Applications," IEEE Trans. Signal Process., vol. 60, no. 11, pp. 5597-5625, 2012.

[2] P. Bianchi, J. Najim, M. Maida, and M. Debbah, "Performance of Some Eigen-based Hypothesis Tests for Collaborative Sensing," IEEE Trans. Inf. Theory, 2010, To appear.

[3] B. Nadler, "Nonparametric Detection of Signals by Information Theoretic Criteria: Performance Analysis and an Improved Estimator," IEEE Trans. Signal Process., vol. 58, no. 5, pp. 2746-2756, 2010.

[4] P. Vallet, P. Loubaton, and X. Mestre, "Improved subspace estimation for multivariate observations of high dimension: the deterministic signals case," IEEE Trans. Inf. Theory, 2010, Submitted for publication.

[5] P. Vallet and P. Loubaton, "A G-estimator of the MIMO channel ergodic capacity," in Proc. IEEE International Symposium on Information Theory (ISIT'09), 2009, pp. 774-778.

[6] A. Kammoun, R. Couillet, J. Najim, and M. Debbah, "Performance of Capacity Inference Methods under Colored Interference," IEEE Trans. Inf. Theory, vol. 59, no. 2, Feb. 2013.

[7] X. Wang and H. V. Poor, "Robust Multiuser Detection in NonGaussian Channels," IEEE Trans. Signal Process., vol. 47, no. 2, Feb. 1999.

[8] E. Contes and M. Lops and G. Ricci, "Asymptotically Optimum Radar Detection in Compound-Gaussian Clutter," IEEE Transactions on Aerospace and Electronic Systems, vol. 31, no. 2, pp. 617-625, 1995.
[9] P. J. Huber, Robust Statistics, Wiley Series in Probability and Statistics John Wiley\& Sons, 1981.

[10] R. A. Maronna, "Robust M-Estimators of Multivariate Location and Scatter," The Annals of Statistics, , no. 4, pp. 51-67, 1976.

[11] R. Couillet and M. McKay, "Large Dimensional Analysis and Optimization of Robust Shrinkage Covariance Matrix Estimators," Journal of Multivariate Analysis, vol. 131, pp. 99-120, 2014.

[12] R. Couillet, F. Pascal, and J. W. Silverstein, "The Random Matrix Regime of Maronna's M-Estimator With Elliptically Distributed Samples," submitted, 2013.

[13] F. Pascal and Y. Chitour and Y. Quek, "Generalized Robust Shrinkage Estimator - Application to STAP Data," Submitted for Publication, 2013, Available: http: //arxiv.org/ pdf/1311.6567.

[14] O. Ledoit and M. Wolf, "A well-conditioned Estimator for Large-Dimensional Covariance Matrices," Journal of Multivariate Analysis, vol. 88, no. 2, pp. 365-411, 2004.

[15] Z. Bai and J. W. Silverstein, "Spectral Analysis of Large Dimensional Random Matrices," Springer Series in Statistics, 2009.

[16] A. Kammoun, M. Kharouf, W. Hachem, and J. Najim, “A Central Limit Theorem for the SINR at the LMMSE Estimator Output for Large Dimensional Signals," IEEE Trans. Inf. Theory, vol. 55, no. 11, Nov. 2009.

[17] R. Couillet and A. Kammoun and F. Pascal, "Second Order Statistics of Robust Estimators of Scatter. Application to GLRT Detection for Elliptical Signals," Submitted to Journal of Multivariate Analysis, 2014. 